\title{
Simultaneous qualitative and quantitative analysis of fluoroalkyl sulfonates in riverine water by liquid chromatography coupled with Orbitrap high resolution mass spectrometry
}

\author{
Yongfeng Lin, Runzeng Liu, Fanbao Hu, Ruirui Liu, Ting Ruan*, Guibin Jiang \\ State Key Laboratory of Environmental Chemistry and Ecotoxicology, Research Center for Eco-Environmental Sciences, Chinese Academy of Sciences, Beijing \\ 100085, China
}

\section{A R T I C L E I N F O}

\section{Article history:}

Received 28 July 2015

Received in revised form

30 December 2015

Accepted 14 January 2016

Available online 16 January 2016

\section{Keywords:}

Perfluoroalkyl sulfonates

Fluorotelomer sulfonates

LC-MS/MS

High resolution mass spectrometry

Tribrid Orbitrap

Riverine water

\begin{abstract}
A B S T R A C T
In this study, a robust method for quick screening, confirmation and quantification analysis of eight fluoroalkyl sulfonates in surface riverine samples was developed using ultra-high performance liquid chromatography-high resolution mass spectrometer (LC-Orbitrap Tribrid HRMS). Weak anion exchange solid phase extraction was optimized to maximum recover perfluoroalkyl sulfonates (PFSAs), fluorotelomer sulfonates and the emerging 6:2 chlorinated polyfluoroalkyl ether sulfonate at the same time. Both qualitative and quantitative purposes could be achieved by simultaneous acquiring full-scan mass spectrum $\left(\mathrm{MS}^{1}\right)$ and data-dependent $\mathrm{MS}^{2}$ data. The LC-Orbitrap Tribrid HRMS method showed competent method detection limits for all analytes (7.1-62 pg/L) compared with the triple quadrupole mass spectrometry (LC-MS/MS) quantification method (12-54 pg/L), and satisfactory method validation results were also obtained in linearity $\left(R^{2}>0.999\right)$, trueness (88-118\%), precision $(2-17 \%)$ and recovery $(63-103 \%)$. A good correlation $(R>0.999)$ was found between the sets of quantified PFSA residue concentrations in thirteen estuary river samples by both the LC-Orbitrap Tribrid HRMS (0.2-440 ng/L) and LC-MS/MS (0.1-424 ng/L) methods, indicating that Orbitrap Tribrid HRMS could be used for reliable quantitative analysis purpose. Moreover, the LC-Orbitrap Tribrid HRMS method could also benefit from its high mass resolution characteristic to eliminate potential environment interferents (e.g., taurodeoxycholate) and to quantify all PFSA isomers based on full-scan $\mathrm{MS}^{1}$ chromatogram at a narrow MS window (5 part per million).
\end{abstract}

(c) 2016 Elsevier B.V. All rights reserved.

\section{Introduction}

Per- and poly-fluoroalkyl sulfonates are a group of anthropogenic chemicals, which possess special physicochemical properties such as hydrophobic and lipophilic balance, and chemical resistance to acid and alkali conditions due to the aggregation of strong carbon-fluorine bonds [1]. The wide usages of the chemicals in industry and daily necessities, such as surfactants, textile, aqueous fire-forming foam, mist suppressant and coatings, have resulted in their inevitable release into the environment since 1950s [2]. Particularly, linear and branched perfluorooctane sulfonate (PFOS) isomers as well as other perfluoroalkyl sulfonate analogs (PFSAs) have become world-wide contaminants due to the persistent and long-range transport characteristics as well as their ubiquitous presence in aqueous environmental com-

\footnotetext{
* Corresponding author. Fax: +86 1062849179

E-mail address: tingruan@rcees.ac.cn (T. Ruan).
}

partments [3-7]. For example, perfluorobutane sulfonate (PFBS), perfluorohexane sulfonate (PFHxS) and PFOS were present with a concentration ranged of $0.94-13.7 \mathrm{ng} / \mathrm{L}$ in Taihu Lake [3]. A range of $0.03-89 \mathrm{ng} / \mathrm{L}$ PFOS was detected in river water of eastern China [4]. In Atlantic, Indian and Pacific oceans, PFBS, PFHxS and PFOS occurred in a range of $20.0-45.4 \mathrm{pg} / \mathrm{L}, 6.65-32.4 \mathrm{pg} / \mathrm{L}$ and $88.5-191 \mathrm{pg} / \mathrm{L}$ respectively [5]. PFOS was also found in Tibetan Mountain with a concentration range of 25.0-64.2 pg/L in surface snow and 61.4-346 pg/L in snow core [6]. Therefore, PFOS and its salts have been voluntarily phased-out by its major manufacturers and have been added to list of the Stock Convention on Persistent Organic Pollutants (Annex B, UNEP) [8]. Other polyfluorinated substances, such as 6:2 chlorinated polyfluorinated ether sulfonate (6:2 Cl-PFAES) and fluorotelomer sulfonates (FTSs) were also found in aqueous systems due to alternative usages $[9,10]$.

The most frequently used instrumental method for PFSA analysis is high performance liquid chromatography coupled to quadrupole tandem mass spectrometry with negative electrospray ion source (HPLC-ESI-MS/MS). Generally, quantitative analysis is 
performed by monitoring the transition between precursor ion and one of the fragmentation ions, while analyte identification is conducted by verifying the ratio of two fragmentation ions. The LCESI-MS/MS methods are competent for the trace analysis of PFSAs with low limits of detection (LODs) in a range of $0.06-1000 \mathrm{pg} / \mathrm{L}$ [11], which covers the residue levels of PFSA homologues in various kinds of environmental medium [5,12,13]. However, accurate quantitative analysis of total PFOS isomers ( $\sum$ PFOS) by the LCESI-MS/MS method is still a challenge. Special columns and long separation time are needed to gain a full isolation of all PFOS isomers. Distinct fragmentation efficiencies of $499 \rightarrow 99$ or $499 \rightarrow 80$ transitions for individual PFOS isomers demand corresponding isotope-labeled standards for accurate quantitation of all PFOS isomers, which are not currently commercial available.

A sensitive liquid chromatography coupled to atmospheric pressure photoionization mass spectrometry (LC-APPI-MS) method was also developed for PFOS analysis with little matrix effect observed (recoveries: 94-97\%) for river water samples [14]. However, the method was only feasible to analytes with low ionization potentials and the corresponding LODs (e.g., PFOS, $5350 \mathrm{pg} / \mathrm{L}$ ) were several times higher compared with those from the LC-ESI-MS/MS method [15]. Single quadrupole HPLC-MS was another sensitive technique developed for PFSA quantitative analysis, but its selectivity was poor for complex matrixes and is thus invalid for qualitative analysis [16]. A novel method for the characterization of PFOS isomers present in technical products was developed using gas chromatography combined with high resolution mass spectrometry (GC-HRMS), however, an additional 2-propanol derivatization step under acidic conditions was needed to increase the volatility of the polyfluorinated anions [17].

Accurate mass measurement and characteristic fragmentation of high resolution mass spectrometer are powerful tools for the elucidation of the molecular structure of emerging environmental contaminants [18]. Novel isopropyl-branched fluorinated substances were initially found in river water by selected suspect-ion monitoring strategy [19], and non-target polyfluorinated sulfonic acid were identified in firefighter serum samples by retrospective screening of the full-scan $\mathrm{MS}^{1}$ spectrum with the aid of advanced statistical analysis [20,21]. Nevertheless, additional LC-MS/MS methods were applied thereafter to quantify the emerging pollutants due to the concern of poor sensitivity and linear dynamic range of HRMS for quantitative purpose. However, this limitation has been overcome due to the synchronous full-scan $\mathrm{MS}^{1}$ and MS/MS acquiring capabilities, which showed advantages on both confirmation and quantification of pesticides and drugs of abuse in various kinds of environmental compartments with the development of hybrid instruments such as Orbitrap and Quadrupole Time-of-Flight, improving cost-effectiveness in the analysis procedures [22-26]. Orbitrap Tribrid HRMS combines the technologies of quadrupole, linear ion trap and orbitrap. The separation of ion isolation, fragmentation and detection could be achieved by Tribrid Orbitrap spatially and temporally compared with the other orbitrap instruments with increased data acquisition rates [27]. To our knowledge, similar application of Tribrid Orbitrap instrument on the simultaneous qualitative and quantitative analysis of polyfluoroalkyl substances has not been conducted.

In the present work, analytical methodology based on the use of solid phase extraction followed by LC-Orbitrap Tribrid HRMS was developed for the determination of PFSAs, FTSs and the emerging 6:2 Cl-PFAES in riverine water. Both full-scan $\mathrm{MS}^{1}$ and $\mathrm{MS}^{2}$ fragmentation information was gathered simultaneously, and quantitative performances of the HRMS analysis method were thoroughly validated compared with those of the routine LC-MS/MS strategy. Specific features of the HRMS method on the effective elimination of plausible analyte interferent and accurate measurement of PFSA isomers were further investigated.

\section{Materials and methods}

\subsection{Chemicals and reagents}

Information on the chemical name, acronym and structure of all analytes is summarized in Table S1. PFBS, PFHxS, PFOS, PFDS, 4:2 FTS, 6:2 FTS, 8:2 FTS, M3PFHxS, MPFOS, and M2-6:2 FTS were purchased from Wellington Laboratories Inc. (Guelph, Ontario). Sodium taurodeoxycholate hydrate was purchased from Sigma-Aldrich (St. Louis, MO). 6:2 Cl-PFAES was obtained from Shanghai Synica Co., Ltd. (Shanghai, China). HPLC grade methanol and ammonium hydroxide $\left(\mathrm{NH}_{4} \mathrm{OH}, 50 \%, \mathrm{v} / \mathrm{v}\right)$ were purchased from J. T. Baker (Phillipsburg, NJ) and Alfa Aesar (Ward Hill, MA), respectively. HPLC grade acetic acid $\left(\mathrm{CH}_{3} \mathrm{CO}_{2} \mathrm{H}\right)$ and ammonium acetate $\left(\mathrm{CH}_{3} \mathrm{CO}_{2} \mathrm{NH}_{4}\right)$ were obtained from DikmaPure (Lake Forest, CA). Ultrapure water was from a Milli-Q water purification system (Millipore, Billerica, MA). $\mathrm{NH}_{4} \mathrm{OH}(0.1 \%, \mathrm{v} / \mathrm{v})$ and $25 \mathrm{mM}$ $\mathrm{CH}_{3} \mathrm{CO}_{2} \mathrm{H} / \mathrm{CH}_{3} \mathrm{CO}_{2} \mathrm{NH}_{4}$ buffer $(\mathrm{pH}=4)$ solutions were prepared in methanol and ultrapure water, respectively. All standard solutions were stored at $4{ }^{\circ} \mathrm{C}$

\subsection{Sample collection and pretreatment}

Approximately $1 \mathrm{~L}$ of each water sample from thirteen main rivers in east cities of China was collected in August, 2013. Sampling sites were near estuary about three meters away from the bank and half a meter below surface. Polypropylene bottles were used as containers, which were pre-washed by methanol and river water for three times. Ultrapure water transported from laboratory was exposed near the river at the sampling period for about $10 \mathrm{~min}$ as the field blank to cover all possible contaminations including containers, sampling and transportation. All water samples were sealed, transported back immediately and stored at $4{ }^{\circ} \mathrm{C}$ until analysis.

Before extraction, all vessels and cartridges were pre-washed by methanol for three times to avoid plausible contamination. All water samples were passed through GF/F glass microfiber filters to remove solid particles, after which $200 \mathrm{~mL}$ of water samples were spiked with mixed internal standards at a concentration of $10 \mathrm{ng} / \mathrm{L}$. M3PFHxS was the internal standard for PFBS and PFHxS, MPFOS was for PFOS, PFDS and 6:2 Cl-PFAES, while M2-6:2 FTS was used for 4:2 FTS, 6:2 FTS and 8:2 FTS. Different solid phase extraction (SPE) materials including mono-functional silane bonded octadecyl (C18, Waters Sep-Pak Vac, $500 \mathrm{mg}, 6 \mathrm{~mL}$ ), hydrophilic-lipophilic balance polymer (HLB, Waters Oasis, $500 \mathrm{mg}, 6 \mathrm{~mL}$ ) and weak anion-exchange polymer (WAX, Waters Oasis, $500 \mathrm{mg}, 6 \mathrm{~mL}$ ) were compared for the extraction of fluoroalkyl sulfonate analytes [11]. C18 and HLB cartridges had the same processing procedures. Both cartridges were firstly rinsed by $4 \mathrm{~mL}$ methanol and $4 \mathrm{~mL}$ ultrapure water, then the water samples were loaded onto the cartridges at a rate of $5-6 \mathrm{~mL} / \mathrm{min}$ for analyte adsorption. The cartridges were dried under vacuum for about $30 \mathrm{~min}$ and finally eluted with $8 \mathrm{~mL}$ methanol. A different processing procedure was applied for WAX cartridge pretreatment according to previous studies [28,29], which used $4 \mathrm{~mL}$ methanol, $4 \mathrm{~mL}$ alkaline methanol $\left(0.1 \% \mathrm{NH}_{4} \mathrm{OH}\right)$ and $4 \mathrm{~mL}$ ultrapure water as the successive rinsing solutions. After loading of samples, the cartridge was washed with methanol and $4 \mathrm{~mL}$ acidized $25 \mathrm{mM} \mathrm{CH}_{3} \mathrm{CO}_{2} \mathrm{H} / \mathrm{CH}_{3} \mathrm{CO}_{2} \mathrm{NH}_{4}$ buffer. Finally, the cartridge was dried under vacuum for about $30 \mathrm{~min}$ and the analytes were eluted by $4 \mathrm{~mL}$ alkaline methanol solution $\left(0.1 \% \mathrm{NH}_{4} \mathrm{OH}\right)$.

All SPE extracts were first evaporated to less than $1 \mathrm{~mL}$ in polypropylene tubes, transferred to glass vials and dried under a gentle nitrogen flow. After that, solution exchange was made by $1 \mathrm{~mL}$ solvent (methanol: $\mathrm{H}_{2} \mathrm{O}=7: 3$ ) added into the vials and mixed sufficiently. An aliquot of $5 \mu \mathrm{L}$ sample extract was injected for 


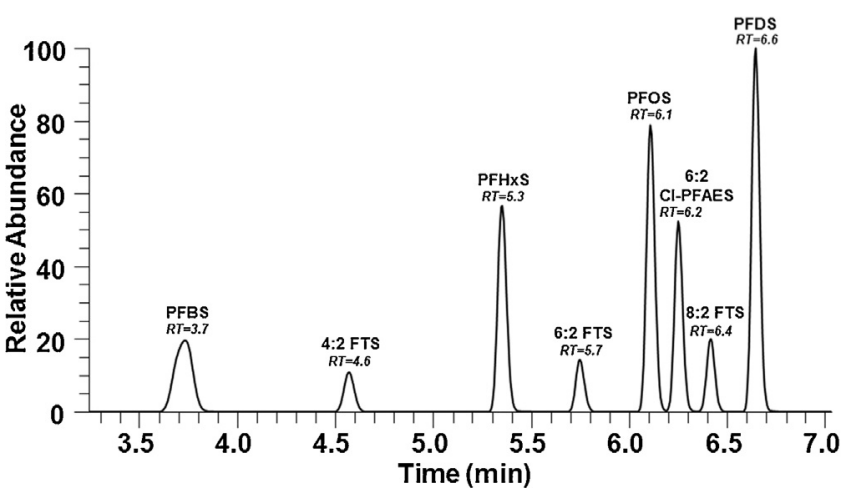

Fig. 1. Retention behaviors of all target analyte standards on the separation column by the LC-Orbitrap Tribrid HRMS method.

further instrument analysis. All samples and standard solutions were sealed and stored at $4{ }^{\circ} \mathrm{C}$ in refrigerator before injection.

\subsection{Instrumentation}

\subsubsection{Liquid chromatography-Orbitrap Tribrid HRMS}

An ACQUITY UPLC C18 column (Waters, $1.7 \mu \mathrm{m}, 2.1 \times 100 \mathrm{~mm}$ ) was used to gain a chromatographic separation of all analytes. The flow rate was $0.3 \mathrm{~mL} / \mathrm{min}$ and column temperature was set at $35^{\circ} \mathrm{C}$. Baseline separation was achieved using mobile phase consisting of methanol and ultrapure water under multi-step gradient elution with a total runtime of $7.0 \mathrm{~min}$ (Fig. 1), and $1 \mathrm{mM}$ ammonium acetate was added into both mobile phases. The initial ratio of methanol was $40 \%$, maintained for $1.0 \mathrm{~min}$, after which the ratio of methanol increased linearly to $100 \%$ in $5.0 \mathrm{~min}$ and kept for another $2 \mathrm{~min}$. After that, the ratio of methanol was diminished to $40 \%$ instantly and kept up for 5 min (Table S2).

The qualification and quantification analysis were performed under negative heated electrospray-ionization (HESI) in full scan $\mathrm{MS}^{1} /$ data-dependent $\mathrm{MS}^{2}$ (dd-MS ${ }^{2}$ ) mode by UltiMate 3000 LCOrbitrap Tribrid HRMS system (Thermo Scientific, Waltham, MA). General instrumental parameters were as follows: Spray voltage, $-2500 \mathrm{~V}$; Ion transfer tube temperature, $350^{\circ} \mathrm{C}$; Vaporizer temperature, $200^{\circ} \mathrm{C}$. Under the full scan $\mathrm{MS}^{1} / \mathrm{dd}^{-\mathrm{MS}^{2}}$ mode, Orbitrap Tribrid HRMS first run a full scan and then followed by a dd-MS ${ }^{2}$ scan. The full scan mass range was set at $200-700 \mathrm{~m} / \mathrm{z}$ with a resolution of 120,000 (FWHM at $200 \mathrm{~m} / \mathrm{z}$ ). Automatic gain control (AGC) target was assigned a value of $1.0 \mathrm{e}^{5}$ (maximum number of ions filling C-Trap) with a maximum injection time of $100 \mathrm{~ms}$. For dd-MS ${ }^{2}$ scan, precursor ion selection range was limited at $250-650 \mathrm{~m} / \mathrm{z}$ with a mass tolerance of $0.5 \mathrm{~m} / \mathrm{z}$ and quadrupole isolated the precursor ions with an isolation window of $1 \mathrm{~m} / \mathrm{z}$. Only those precursor ions that had a signal threshold $>1.0 \mathrm{e}^{4}$ counts were permissible for $\mathrm{MS}^{2}$ fragmentation activation with $40 \%$ collision energy. The orbitrap resolution was set at 15,000 FWHM, AGC target at $5.0 \mathrm{e}^{4}$ for dd$\mathrm{MS}^{2}$ scan period. Xcalibur (Thermo Scientific, USA) was used for both qualitative and quantitative analysis.

\subsubsection{Triple quadrupole mass spectrometry}

An UltiMate 3000 LC coupled with a Triple Quad 5500 (AB SCIEX, Framingham, MA) mass analyzer was utilized for comparative quantification analysis. Qualification and quantification of target analytes were achieved in negative electrospray ionization MRM (multiple reaction monitoring) mode in which nitrogen was used as the ion source gas, curtain gas and collision gas. Analyst Software (AB SCIEX, California, USA) was used for data analysis.

\subsection{Method validation}

Method validation covered most of the key points such as selectivity, linearity, method detection limit (MDL), method quantitation limit (MQL), trueness, precision, recovery and matrix effect.

Selectivity describes whether analytical method could discriminate interference of similar compounds, which was determined by comparison of both the chromatogram retention time (RT) and accurate mass from the Orbitrap HRMS. For linearity test, a ninepoint curve $(0.1,0.2,0.5,1.0,2.0,5.0,10,20$ and $50 \mathrm{ng} / \mathrm{mL})$ was fitted using standard solution of all analytes. Method quantification limits of the Orbitrap Tribrid HRMS method were calculated according to equation 1 and 2 as mentioned elsewhere [30,31]

$$
\begin{aligned}
& \mathrm{MDL}=A+t \times S \\
& \mathrm{MQL}=3 \times \mathrm{MDL}
\end{aligned}
$$

in which $A$ was the intercept of $\mathrm{X}$-axis calculated from the fitted regression equation at five low concentration levels $(0.01,0.02$, $0.05,0.08$ and $0.12 \mathrm{ng} / \mathrm{mL}$ ). $S$ was the standard deviation of replicate analysis of blank water samples $(200 \mathrm{~mL})$ spiked with the mixed standards at a concentration of $1.0 \mathrm{ng} / \mathrm{L}(\mathrm{n}=5)$. $t$ was the obtained from the $t$-test confidence level $\left(t_{n=5, p=0.01}=3.4\right)$.

Trueness and precision are two important characteristics of instrument stability, where trueness describes the reliability and precision depicts the repeatability of the optimized method. Trueness was calculated as the relative error between the determined and reference value of standard samples, and precision was measured as the relative standard deviation of repeat quantitative analysis of the same sample at different time period. The intraday and inter-day precision of both LC-Orbitrap Tribrid HRMS and LC-MS/MS methods were assessed with three repetitions of a blank water sample per spiking levels (1, 10 and $100 \mathrm{ng} / \mathrm{L}$ ), which were repeated in four consecutive days.

Recovery characterizes the mass loss of analytes during the whole analytical procedure, which was determined by peak area ratios of analytes spiked into independent blank water samples ( $n=4$ in each of 1,10 , and $100 \mathrm{ng} / \mathrm{L}$ spiking levels) before and after extraction. Matrix effect was calculated by comparing the response of standard analytes spiked in extracted eluent of blank water samples and in pure solvent, which was also assessed at the three concentration levels and repetitions as in the recovery tests. The recoveries were all calculated by the LC-Orbitrap Tribrid HRMS method.

\section{Results and discussion}

\subsection{Instrument parameter optimization}

Fragmentation ions in the MS ${ }^{n}$ spectrum provide critical information on elucidating chemical structures of analytes, which could also be used for quantification purpose. Distinct fragmentation patterns of PFSA analogs were optimized in both collision induced dissociation (CID) and higher-energy collisional dissociation (HCD) modes to acquire characteristic fragment ion information.

As shown in Fig. 2, diverse $\mathrm{MS}^{2}$ ions were observed for individual fluoroalkyl sulfonate analogs in different fragmentation mode. For example, the main daughter ions of PFSAs were $\left[\mathrm{SO}_{3}\right]^{-}$, $\left[\mathrm{FSO}_{3}\right]^{-}$in HCD mode and $\left[\mathrm{C}_{n} \mathrm{~F}_{2 n+1}\right]^{-},\left[\mathrm{C}_{n} \mathrm{~F}_{2 n} \mathrm{SO}_{3}\right]^{-}(n=3-6)$ in $\mathrm{CID}$ mode. Besides the preferred fragmentation pathway to give $\left[\mathrm{SO}_{3}\right]^{-}$and $\left[\mathrm{FSO}_{3}\right]^{-}$at $\mathrm{m} / \mathrm{z} 80$ and 99 [32], the observed cleavage patterns involving a remote charge fragmentation mechanism to generate a series of $\left[\mathrm{C}_{n} \mathrm{~F}_{2 n} \mathrm{SO}_{3}\right]^{-}$anions as well as homolytic unzipping $\left[\mathrm{C}_{n} \mathrm{~F}_{2 n+1}\right]^{-}$ions due to fluorine migration effect after initial loss of $\mathrm{SO}_{3}$ were thoroughly investigated in previous studies [33]. Obvious differences were also found between HCD and 
PFHxS@HCD
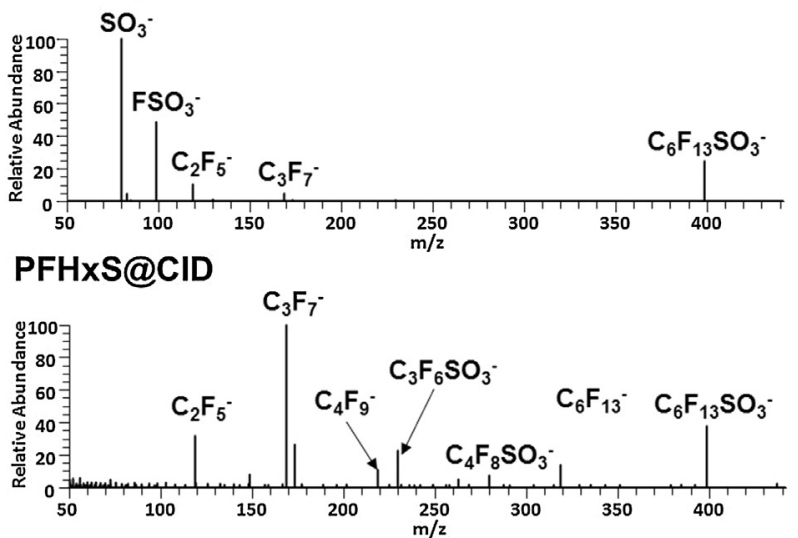

(A)

\section{6:2 FTS@HCD}

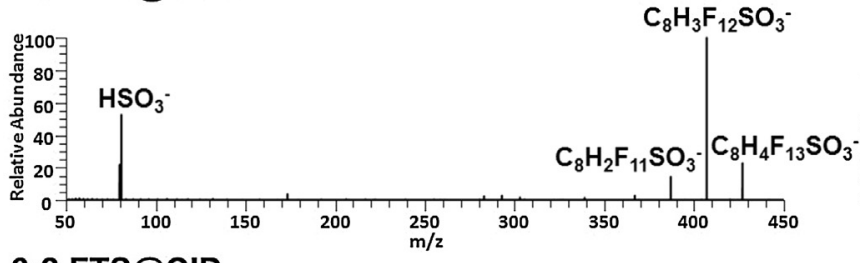

6:2 FTS@CID

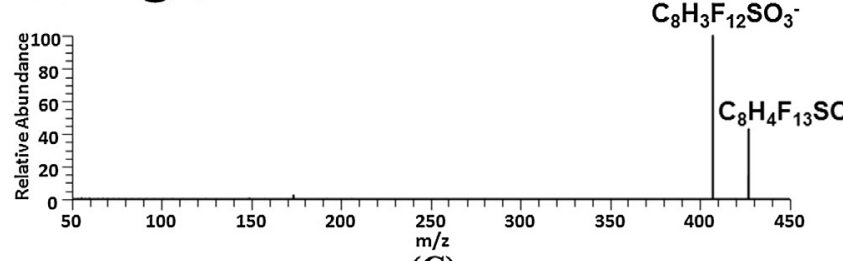

(C)
PFOS@HCD

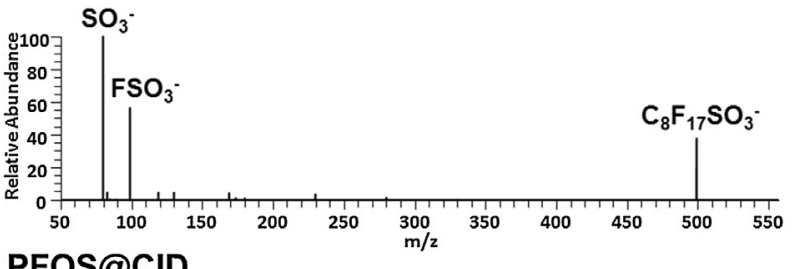

PFOS@CID

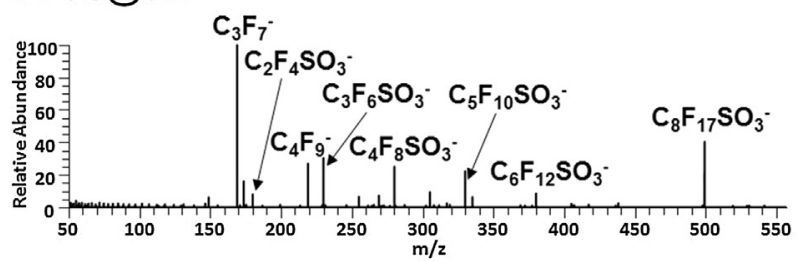

(B)

\section{6:2 CI-PFAES@HCD}



6:2 CI-PFAES@CID

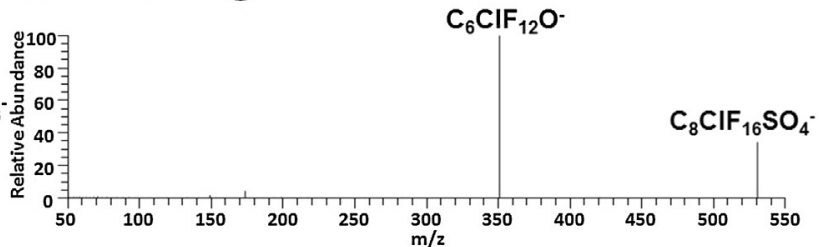

(D)

Fig. 2. Data dependent-MS² fragmentation ions of target analytes (A. PFHxS, B. L-PFOS, C. 6:2 FTS, D. 6:2 Cl-PFAES) in both HCD and CID modes.

CID fragmentation, which could be influenced by many factors. For instance, HCD is more drastic compared with CID that more low mass fragmentation ions could be obtained. Also, CID suffers from a low-mass cutoff that monitoring the low mass fragmentation ions such as $\left[\mathrm{SO}_{3}\right]^{-}$and $\left[\mathrm{FSO}_{3}\right]^{-}$were not feasible. Meanwhile, distinguished characteristic $\mathrm{MS}^{2}$ ions were noticed for other analytes. The major fragmentation ions of $\left[\mathrm{C}_{n+2} \mathrm{H}_{2} \mathrm{~F}_{2 n-1} \mathrm{SO}_{3}\right]^{-},\left[\mathrm{HSO}_{3}\right]^{-}$ in $\mathrm{HCD}$ mode and $\left[\mathrm{C}_{n+2} \mathrm{H}_{3} \mathrm{~F}_{2 n} \mathrm{SO}_{3}\right]^{-}$in CID mode $(n=4,6,8)$ were found for FTSs. While, $\left[\mathrm{C}_{6} \mathrm{ClF}_{12} \mathrm{O}\right]^{-}$, $\left[\mathrm{FSO}_{2}\right]^{-}$in $\mathrm{HCD}$ mode and only $\left[\mathrm{C}_{6} \mathrm{ClF}_{12} \mathrm{O}\right]^{-}$in CID mode were obtained for 6:2 Cl-PFAES. Considering more abundant information at low-mass range by HCD mode, it was selected as the activation type in full scan MS $^{1} /$ dd-MS $^{2}$ experiments.

Moreover, a pump-driven injection was processed using standard solutions to ensure appropriate HCD collision energy for each precursor ion, so that enough fragmentation ions could be generated in $\mathrm{MS}^{2}$ scanning. Take 6:2 Cl-PFAES as an example, at a lower HCD energy of $20 \%$, few fragmentation ions could be detected. Along with the rise of the HCD energy, the relative abundance of fragmentation ions $\left(\left[\mathrm{C}_{6} \mathrm{ClF}_{12} \mathrm{O}\right]^{-}\right.$and $\left.\left[\mathrm{FSO}_{2}\right]^{-}\right)$increased obviously. When the HCD energy was set as $50 \%$, the precursor ions would absolutely crushed into the fragmentation ions (Fig. S1).

For LC-MS/MS, a pump-driven injection experiment was also conducted to optimize declustering potential (DP) and collision energy (CE) in MRM acquisition. The data showed that the DPs were almost similar for each analyte, while CE increased apparently as the chain length grew. Detailed information was presented in Table S3.

\subsection{Sample pretreatment optimization}

SPE is the mostly used pretreatment strategy for analyte extraction and concentration in aqueous samples, and various SPE methods have been developed for PFSA analysis using various kinds of adsorption materials [34]. Factors with the most significant impacts on SPE efficiency, including type of cartridges, cartridge capacity, washing buffer ratio and eluent volume, were optimized as follows.

\subsubsection{Type of SPE cartridge}

Three frequently used SPE cartridges (Sep-Pak C18, Oasis HLB, Oasis WAX) were compared to determine extraction performances for the fluoroalkyl sulfonate analytes [34]. As listed in Fig. S2, PFBS displayed low retention in C18 (recovery: $26 \pm 5 \%$ ) and HLB cartridges $(52 \pm 8 \%$ ) compared with that in WAX tubes $(113 \pm 17 \%)$. The results suggest that reversed-phase adsorption mechanism might be insufficient to retain short-chain PFBS molecules, but the tertiary amine functional group in the WAX adsorbent showed satisfactory interaction with the polyfluorinated weak anions.

At the same time, all three cartridges exhibited unsatisfactory recoveries ( $60 \%$ ) for PFDS, which might be due to its long chain strongly intertwined with the sorbents, making it hard to elute. For other analogs such as PFHxS, linear PFOS isomer (L-PFOS), FTSs and 6:2 Cl-PFAES, the three kinds of cartridges all showed satisfactory recoveries in the range of $82-122 \%(n=3)$ (Fig. S2.A). The distinct retention behaviors associated with PFSA carbon-chain length have been universally reported for C18, HLB and WAX cartridges [28,35]. Nevertheless, satisfactory recoveries found in this study also 




(A)

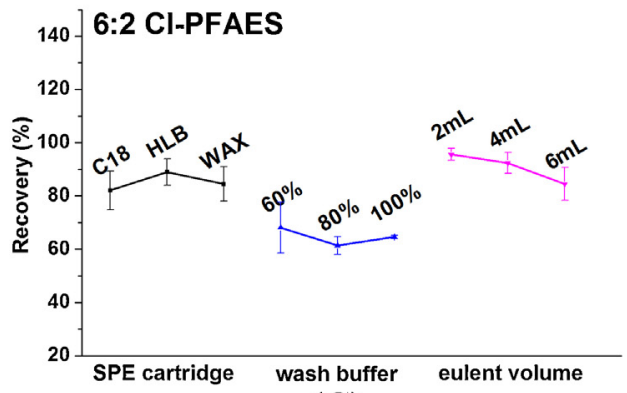

(C)

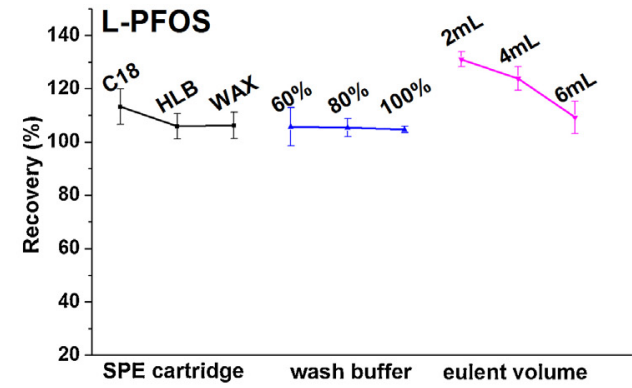

(B)

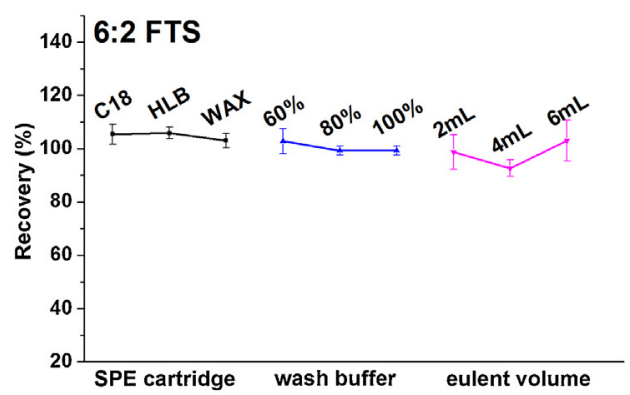

(D)

Fig. 3. Performances of influence factors on SPE pretreatment procedures for (A) PFBS, (B) L-PFOS, (C) 6:2 Cl-PFAES and (D) 6:2 FTS.

suggest applicability of the SPE pretreatment for the emerging 6:2 Cl-PFAES chemical. As WAX cartridge showed better selectivity for short-chain weak anion compounds, it was selected for the following experiments.

\subsubsection{Cartridge capacity}

Generally, each SPE cartridge has an upper adsorption limit for individual adsorbates, beyond which the cartridge will lose the adsorbability for analytes. The capacity of Oasis WAX cartridges was tested at three concentration levels (2, 20 and $200 \mathrm{ng} / \mathrm{L})$. As presented in Fig. S2.C, no significant difference was found for PFSAs, FTSs and 6:2 Cl-PFAES ( $t$-test, $p>0.05$ ) with corresponding recoveries in the range of $48-113 \%, 62-119 \%, 58-85 \%$ respectively. The standard deviation results at the three concentration levels were in a range of 4-20\% for all analytes except for 6:2 FTS with a value of $28 \%$. In brief, the results indicate the WAX cartridge could absorb all analytes even at a high total mount ( $40 \mathrm{ng}$ of individual analyte per column), which is competent for the analysis of real water samples.

\subsubsection{Methanol/water washing buffer ratio}

Washing buffer with different methanol proportions were checked at three values $(60,80$ and $100 \%)$ in order to maximum eliminate possible interferents. The average recoveries in the three conditions were all in a range of $47-105 \%$ for PFSA analogs, with deviations ranged from $1 \%$ (L-PFOS) to $10 \%$ (PFBS). For FTS analogs, the average recoveries ranged from $100 \%$ to $127 \%$, with deviations in a range of $1-8 \%$. The average recovery for $6: 2$ Cl-PFAES was $65 \%$, where the deviation was minor (3\%) (Fig. S2.B). In comparison with the other two washing buffer ratios, the recoveries of all analytes showed negligible decrease at $100 \%$ methanol proportion. Thus, $4 \mathrm{~mL}$ of methanol was finally used as the washing buffer.

\subsubsection{Volume of eluent}

Alkaline methanol containing $0.1 \% \mathrm{NH}_{4} \mathrm{OH}$ was further used to neutralize and to elute the fluoroalkyl sulfonates from the WAX columns. The eluent volume ( $2 \mathrm{~mL}, 4 \mathrm{~mL}$ and $6 \mathrm{~mL}$ ) was optimized in order to recovery target analytes as much as possible. The recoveries of PFSAs were similar at $2 \mathrm{~mL}, 4 \mathrm{~mL}$ and $6 \mathrm{~mL}$ eluent, and the same phenomenon were found for 6:2 Cl-PFAES and FTSs, with all recoveries in the range of $85-115 \%$ (Fig. S2.D). Thus, $4 \mathrm{~mL}$ of solvent was chosen as the optimized eluent volume.

\subsubsection{Performances of influence factors on SPE}

The performances of parameter optimization, including type of adsorbents, washing buffer ratio and eluent volume, on the recoveries of several representative analytes were further compared. In Fig. 3, the vertical distance between the highest and lowest plot expresses the recovery improvement for each factor during SPE processes. For PFBS, type of adsorbents was the most vital factor with a recovery changed from $26 \%$ (C18) to $113 \%$ (WAX), while the other two factors had little influence on the recovery. No significant differences of recovery were found for the other three analytes including L-PFOS, 6:2 Cl-PFAES and 6:2 FTS. In summary, 6:2 Cl-PFAES and the FTS analogs showed similar retention behaviors compared with the PFSAs ( $\mathrm{C} 6, \mathrm{C} 8, \mathrm{C} 10)$ during pretreatment processes, indicating that the SPE method was applicable to the emerging polyfluorinated substances.

\subsection{Method validation}

\subsubsection{Selectivity}

Analytes were confirmed based on the retention time and accurate mass deviation in the Orbitrap Tribrid HRMS . Retention time remained stable during the whole experiment in both standard solutions and water samples. A mass deviation of less than $1 \mathrm{ppm}$ (part-per-million) for precursor ions and $4 \mathrm{ppm}$ for fragmentation ions was obtained (Table S3). The extracted ion chromatograms of all monitored ions in blank water samples at the mass tolerance window of $1 \mathrm{ppm}, 5 \mathrm{ppm}$ and $10 \mathrm{ppm}$ were examined to check whether possible interferences could be effectively excluded in the suspect screening analysis. Fig. S3 showed that only one interference was found in the extracted PFDS ion chromatogram at $10 \mathrm{ppm}$ mass tolerant window, the retention time of which (RT: $6.89 \mathrm{~min}$ ) was close to that of the PFDS standard (RT: $6.65 \mathrm{~min})$. 
Table 1

Comparison of linearity, MDL and MQL between the Orbitrap Tribrid HRMS and LC-MS/MS methods.

\begin{tabular}{|c|c|c|c|c|c|c|}
\hline \multirow[t]{2}{*}{ Acronym } & \multicolumn{2}{|c|}{ Linearity $/ R^{2}$} & \multicolumn{2}{|c|}{ MDL (pg/L) } & \multicolumn{2}{|c|}{$\mathrm{MQL}(\mathrm{pg} / \mathrm{L})$} \\
\hline & Orbitrap & $\mathrm{MS} / \mathrm{MS}$ & Orbitrap & MS/MS & Orbitrap & MS/MS \\
\hline PFBS & 0.9995 & 0.9994 & 36 & 24 & 107 & 71 \\
\hline PFHxS & 0.9996 & 0.9997 & 24 & 28 & 71 & 83 \\
\hline PFOS & 0.9992 & 0.9998 & 24 & 16 & 73 & 49 \\
\hline PFDS & 0.9990 & 0.9998 & 23 & 31 & 70 & 93 \\
\hline $6: 2$ Cl-PFAES & 0.9993 & 0.9998 & 7.1 & 12 & 21 & 35 \\
\hline $4: 2$ FTS & 0.9994 & 0.9993 & 59 & 47 & 177 & 140 \\
\hline $6: 2$ FTS & 0.9993 & 0.9986 & 62 & 41 & 187 & 122 \\
\hline $8: 2$ FTS & 0.9997 & 0.9982 & 54 & 54 & 161 & 163 \\
\hline
\end{tabular}

An identification point (IP) evaluation system mentioned elsewhere was also introduced for analyte confirmation in the LC-Orbitrap Tribrid HRMS and LC-MS/MS methods with little modification [36,37]. In brief, at least 3.0 IPs should be possessed for positive confirmation of environmental contaminants. For HRMS, 2.0 IPs could be earned by monitoring precursor ion, with another 2.5 IPs for each fragmentation ion. In full scan $\mathrm{MS}^{1} / \mathrm{dd}^{-\mathrm{MS}^{2}}$ mode, one precursor ion and one fragmentation ion could be acquired for each analyte. Thus, a total of 4.5 IPs were gained, which was sufficient for analyte confirmation. With regards to LC-MS/MS, 1.0 IPs could be earned for one precursor ion in unit resolution, with 1.5 IPs for each fragmentation ion. One precursor ion and two fragmentation ions were acquired in the MRM experiments, so that a total of 4.0 IPs could be obtained.

Taurodeoxycholate (TDCA, $\left[\mathrm{C}_{26} \mathrm{H}_{44} \mathrm{NO}_{6} \mathrm{~S}\right]^{-}$) was recognized as an interferent in LC-MS/MS analysis, which could co-elute with PFOS on C18 column [38]. Also, TDCA could induce the same transition of $499 \rightarrow 80\left([\mathrm{M}]^{-} \rightarrow\left[\mathrm{SO}_{3}\right]^{-}\right)$in unit resolution as that for PFOS quantification purpose, which might thus bring in false positive results. However, the interference would not happen in the HRMS analysis due to the high resolution power of orbitrap. As shown in Fig. S4, the instrument measuring mass errors of TDCA (accurate mass: $m / z=498.2902$, mass error $=0.7 \mathrm{mDa}$ ) and PFOS $(m / z=498.9306$, mass error $=0.4 \mathrm{mDa})$ in water samples compared with the individual theoretical molecular weights could be easily distinguished from the significant mass difference of the two compounds ( $\Delta M=640.4 \mathrm{mDa}$ ).

In the LC-MS/MS analysis, accurate measurement of $\sum$ PFOS is challenging due to the varied fragmentation efficiencies of linear and branched isomers. Nevertheless, PFOS precursor ion was used for the quantitative purpose in the Orbitrap Tribrid HRMS method. Ionization efficiencies of L-PFOS and branched PFOS (Br-PFOS) were compared at five spiking concentrations $(0.1,1,5,10$, and $20 \mathrm{ng} / \mathrm{mL})$ $(n=3)$ to ensure the feasibility of this method. Fig. S5 shows that the average relative ionization efficiencies of the $\sum \mathrm{Br}$-PFOS and $\sum$ PFOS precursor ions were $99 \pm 16 \%$ and $99 \pm 21 \%$ compared with that of the L-PFOS. Thus, reliable analysis of $\sum$ PFOS concentrations in environmental samples could be achieved by quantifying the precursor ion in the full scan MS ${ }^{1}$ spectrum, which could be another unique advantage of HRMS quantification strategy compared with the LC-MS/MS method.

\subsection{2. $M D L, M Q L$ and linearity}

Method detection limit (MDL) and method quantification limit (MQL) reflect the lower identification and quantitation level of the method. Similar performance was found for PFSAs, 6:2 Cl-PFAES and FTSs by both instrument methods. The MDLs were $23-36 \mathrm{pg} / \mathrm{L}$, $7.1 \mathrm{pg} / \mathrm{L}$ and $54-62 \mathrm{pg} / \mathrm{L}$ by LC-Orbitrap Tribrid HRMS, respectively, while those for the LC-MS/MS quantification method were $16-31 \mathrm{pg} / \mathrm{L}, 12 \mathrm{pg} / \mathrm{L}$ and $41-54 \mathrm{pg} / \mathrm{L}$, respectively (Table 1 ). Generally, residue concentrations of PFSA analogs in surface water were reported in the range of $0.03-14 \mathrm{ng} / \mathrm{L}$ in China [4], $0.03-5.5 \mathrm{ng} / \mathrm{L}$ in north of America [39], 0.08-181 ng/L in Europe [13], which could be easily covered by both of the Orbitrap Tribrid HRMS and LC-MS/MS trace quantification dynamic ranges $(0.1-50 \mathrm{ng} / \mathrm{mL})$. Also, all analytes were calibrated with good coefficients of determination $\left(R^{2}\right)$ all $\geq 0.9990$ for Orbitrap Tribrid HRMS and $\geq 0.9982$ for LC-MS/MS at $1 / x$ weighting (Table S4).

\subsubsection{Trueness and precision}

Trueness was calculated as a ratio between the average measured concentration $(n=8)$ and the certified one for each analyte at three spiking levels $(1,10$ and $100 \mathrm{ng} / \mathrm{L})$, which is illustrated in Eq. (3).

Trueness $(\%)=\frac{C_{\mathrm{d}} \times 100}{C_{\mathrm{s}}} \%$

$C_{\mathrm{d}}$ was the average measured analyte concentration in quality control samples. $C_{\mathrm{s}}$ was the certified concentration.

Trueness in the range of $88-106 \%$ and $90-101 \%$ were acquired by Orbitrap Tribrid HRMS and LC-MS/MS method, respectively, for all analytes at the concentration of $1 \mathrm{ng} / \mathrm{L}$. The trueness got a range of $102-118 \%, 99-108 \%$ at 10 and $100 \mathrm{ng} / \mathrm{L}$ for Orbitrap Tribrid HRMS strategy, while those of the LC-MS/MS method were 105-127\% and $96-109 \%$, respectively. Precision was described by the standard deviation of repeated injections in a day (intra-day precision) and during four days (inter-day precision). As listed in Table 2, the calculated intra-day and inter-day precision of all analytes were all $<17 \%$ for Orbitrap Tribrid HRMS and $<15 \%$ for LC-MS/MS method at the three spiking concentrations

The trueness and precision results of the Orbitrap Tribrid HRMS method were within the acceptable criteria of $80-120 \%$ for trueness and $<20 \%$ for precision, indicating that the developed method was accurate, believable and repeatable for the analysis of PFSAs in surface water matrix.

\subsubsection{Recovery and matrix effect}

Recovery was evaluated by measuring the analyte residue levels after sample pretreatment in blank water samples with mixed native standards spiked at 1,10 and $100 \mathrm{ng} / \mathrm{L}(n=4$ per each spiking level). For PFSA analogs, PFDS had a relatively low recovery at $100 \mathrm{ng} / \mathrm{L}$ while the other PFSAs showed satisfactory recoveries in the range of $71-103 \%$ at all three levels. For 6:2 Cl-PFAES and FTSs, all the recoveries ranged from $73 \%$ to $95 \%$ at the three spiking levels. The satisfactory recovery results indicated that the optimized sample extraction procedure was competent for the analysis of PFSAs including the emerging 6:2 Cl-PFAES analog.

Matrix effect was characterized by the analyte signal enhancement or suppression resulted from the influence of plausible environment interferent in the sample extract. It was calculated by the ratio of peak areas in matrix spiked solution and standard solution at 1,10 and $100 \mathrm{ng} / \mathrm{L}$ (Eq. (4)).

Matrix effect $(\%)=\frac{A_{\mathrm{m}} \times 100}{A_{\mathrm{s}}} \%$

$A_{\mathrm{m}}$ was the spiked analyte instrument response in blank matrix. $A_{\mathrm{s}}$ was the instrument response in standard solution at the same spiking level.

A matrix effect ranging from $80-120 \%$ is generally considered acceptable. At the low spiking concentration level (1 ng/L), PFBS, PFHxS and 8:2 FTS showed obvious signal enhancement with matrix effects larger than $120 \%$ whereas the other analytes had matrix effects ranging 100-119\% (Table 2). The matrix effects for all analytes decreased to a range of $95-112 \%$ at $10 \mathrm{ng} / \mathrm{L}$, except for 8:2 FTS. At $100 \mathrm{ng} / \mathrm{L}$ level, the measured matrix effects were in a range of $100 \pm 11 \%$ for all analytes. Meanwhile, all target analytes had similar results in LC-MS/MS method that the matrix effects were $96-104 \%$ at all three concentration levels. 
Table 2

Detailed method validation parameters of both the Orbitrap Tribrid HRMS and LC-MS/MS methods.

\begin{tabular}{|c|c|c|c|c|c|c|c|c|c|c|}
\hline \multirow[t]{2}{*}{ Acronym } & \multirow[t]{2}{*}{ Spiked/(ng/L) } & \multicolumn{2}{|c|}{ Trueness (\%) } & \multicolumn{2}{|c|}{ Precision-intra (\%) } & \multicolumn{2}{|c|}{ Precision-inter (\%) } & \multicolumn{2}{|c|}{ Matrix effect (\%) } & \multirow[t]{2}{*}{ Recovery $^{\mathrm{a}}(\%)$} \\
\hline & & Orbitrap & MS/MS & Orbitrap & MS/MS & Orbitrap & MS/MS & Orbitrap & MS/MS & \\
\hline \multirow[t]{3}{*}{ PFBS } & 1 & 98 & 100 & 9 & 9 & 12 & 13 & $134 \pm 5$ & $100 \pm 3$ & $95 \pm 3$ \\
\hline & 10 & 102 & 116 & 2 & 5 & 3 & 5 & $96 \pm 3$ & $101 \pm 3$ & $103 \pm 3$ \\
\hline & 100 & 105 & 109 & 6 & 5 & 6 & 8 & $93 \pm 2$ & $101 \pm 5$ & $90 \pm 1$ \\
\hline \multirow[t]{3}{*}{ PFHxS } & 1 & 96 & 96 & 8 & 3 & 11 & 7 & $122 \pm 4$ & $100 \pm 6$ & $88 \pm 6$ \\
\hline & 10 & 107 & 109 & 4 & 5 & 5 & 5 & $103 \pm 4$ & $100 \pm 3$ & $90 \pm 3$ \\
\hline & 100 & 100 & 104 & 9 & 8 & 8 & 7 & $100 \pm 1$ & $100 \pm 6$ & $87 \pm 3$ \\
\hline \multirow[t]{3}{*}{ PFOS } & 1 & 98 & 95 & 5 & 8 & 13 & 14 & $100 \pm 12$ & $98 \pm 7$ & $80 \pm 5$ \\
\hline & 10 & 106 & 110 & 3 & 5 & 8 & 11 & $109 \pm 3$ & $100 \pm 8$ & $71 \pm 2$ \\
\hline & 100 & 99 & 101 & 11 & 8 & 11 & 14 & $92 \pm 11$ & $100 \pm 5$ & $78 \pm 5$ \\
\hline \multirow[t]{3}{*}{ PFDS } & 1 & 97 & 101 & 7 & 7 & 12 & 12 & $119 \pm 6$ & $101 \pm 6$ & $76 \pm 4$ \\
\hline & 10 & 106 & 108 & 16 & 15 & 16 & 14 & $101 \pm 2$ & $100 \pm 6$ & $66 \pm 6$ \\
\hline & 100 & 100 & 98 & 11 & 12 & 13 & 15 & $89 \pm 10$ & $100 \pm 6$ & $63 \pm 7$ \\
\hline $6: 2$ & 1 & 88 & 91 & 5 & 9 & 12 & 14 & $111 \pm 16$ & $104 \pm 11$ & $84 \pm 3$ \\
\hline \multirow[t]{2}{*}{ Cl-PFAES } & 10 & 107 & 105 & 2 & 12 & 8 & 9 & $95 \pm 3$ & $101 \pm 14$ & $86 \pm 3$ \\
\hline & 100 & 102 & 98 & 10 & 5 & 14 & 8 & $95 \pm 10$ & $100 \pm 6$ & $85 \pm 4$ \\
\hline $4: 2$ & 1 & 94 & 92 & 10 & 7 & 12 & 10 & $107 \pm 0$ & $96 \pm 9$ & $95 \pm 4$ \\
\hline \multirow{2}{*}{ FTS } & 10 & 105 & 120 & 8 & 5 & 9 & 5 & $112 \pm 2$ & $100 \pm 9$ & $92 \pm 1$ \\
\hline & 100 & 101 & 104 & 8 & 10 & 8 & 8 & $119 \pm 1$ & $100 \pm 6$ & $90 \pm 5$ \\
\hline $6: 2$ & 1 & 99 & 90 & 7 & 3 & 16 & 9 & $108 \pm 4$ & $100 \pm 4$ & $94 \pm 4$ \\
\hline \multirow[t]{2}{*}{ FTS } & 10 & 107 & 120 & 13 & 9 & 15 & 15 & $103 \pm 1$ & $100 \pm 3$ & $93 \pm 0$ \\
\hline & 100 & 99 & 96 & 7 & 6 & 6 & 8 & $101 \pm 2$ & $101 \pm 6$ & $87 \pm 3$ \\
\hline $8: 2$ & 1 & 107 & 93 & 15 & 10 & 17 & 12 & $151 \pm 8$ & $101 \pm 13$ & $82 \pm 6$ \\
\hline \multirow[t]{2}{*}{ FTS } & 10 & 118 & 127 & 8 & 11 & 9 & 8 & $131 \pm 3$ & $99 \pm 5$ & $73 \pm 3$ \\
\hline & 100 & 108 & 101 & 11 & 9 & 11 & 11 & $112 \pm 3$ & $99 \pm 7$ & $73 \pm 2$ \\
\hline
\end{tabular}

a Calculated by the LC-Orbitrap Tribrid HRMS method.

\subsection{Application to water samples}

Thirteen samples collected from the main estuaries of east China were analyzed by both LC-Orbitrap Tribrid HRMS and LC-MS/MS methods. Simultaneous qualification and quantification of analytes were performed in full scan $\mathrm{MS}^{1} / \mathrm{dd}^{-M S^{2}}$ mode by LC-Orbitrap Tribrid HRMS method. Target analyte identification was based on chromatograph separation RT and accurate mass deviation. If there was no signal or signal below MDL at the RT under a mass deviation of $5 \mathrm{ppm}$, the existence of particular analyte in the sample was considered negative. The extracted ion chromatograms and dd-MS ${ }^{2}$ fragmentation ions of detected analytes in water samples are shown in Fig. 4. The retention behaviors of the analytes in water samples were in good accordance with those of the certified standards (Fig. 1), and detected accurate mass deviation for precursor ions and the fragmentation ions were all less than $5 \mathrm{ppm}$. Quantitation of analytes was performed by precursor ions during full mass scan stage. PFBS, PFHxS, L-PFOS, 6:2 Cl-PFAES were detected in all samples at concentrations ranging $0.5-440 \mathrm{ng} / \mathrm{L}, 0.3-17.0 \mathrm{ng} / \mathrm{L}, 1.9-14.5 \mathrm{ng} / \mathrm{L}$ and $2.0-44.2 \mathrm{ng} / \mathrm{L}$ respectively. No 4:2 FTS and 8:2 FTS was detected in the samples. PFDS was only detected in Yellow river at a

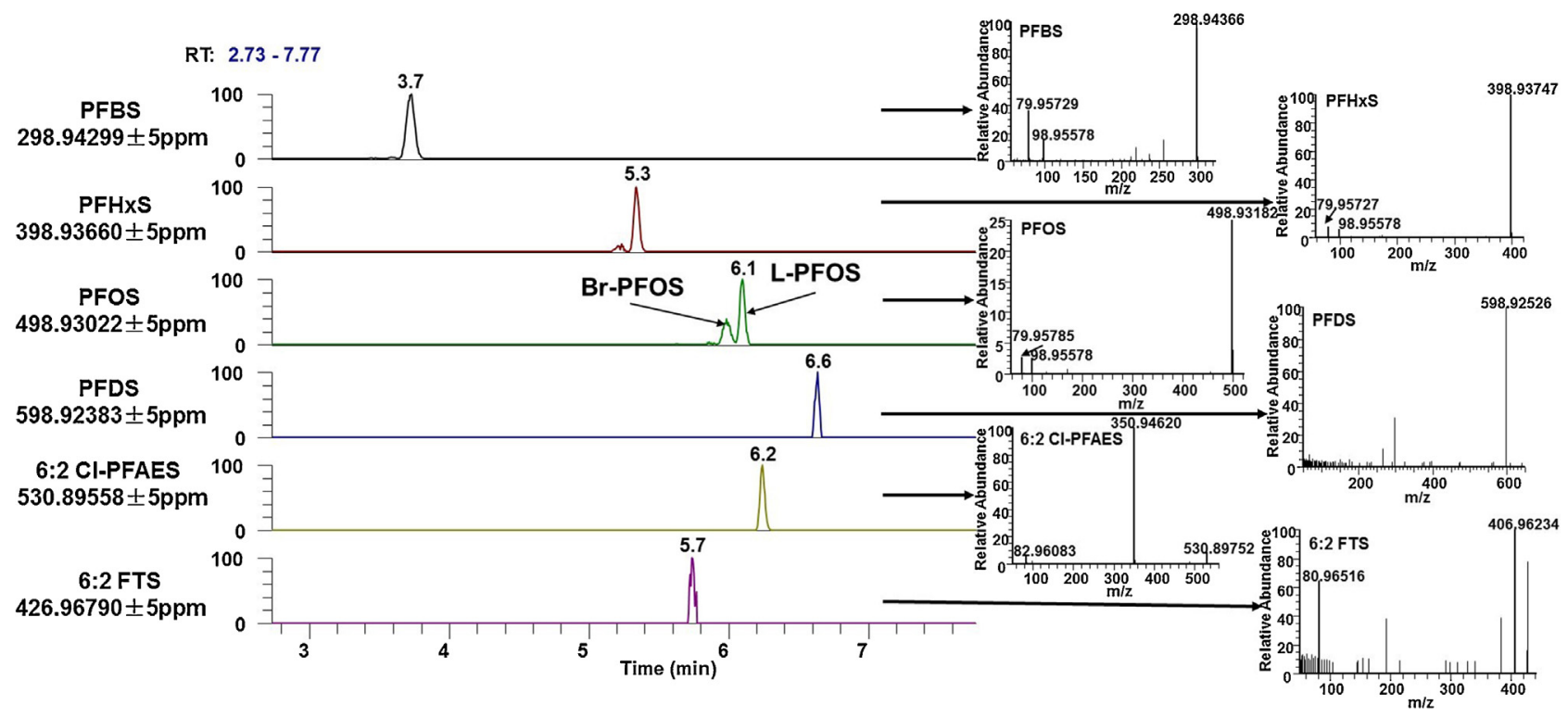

Fig. 4. Retention behaviors and dd-MS ${ }^{2}$ fragmentation ions of identified analytes in the water samples. 


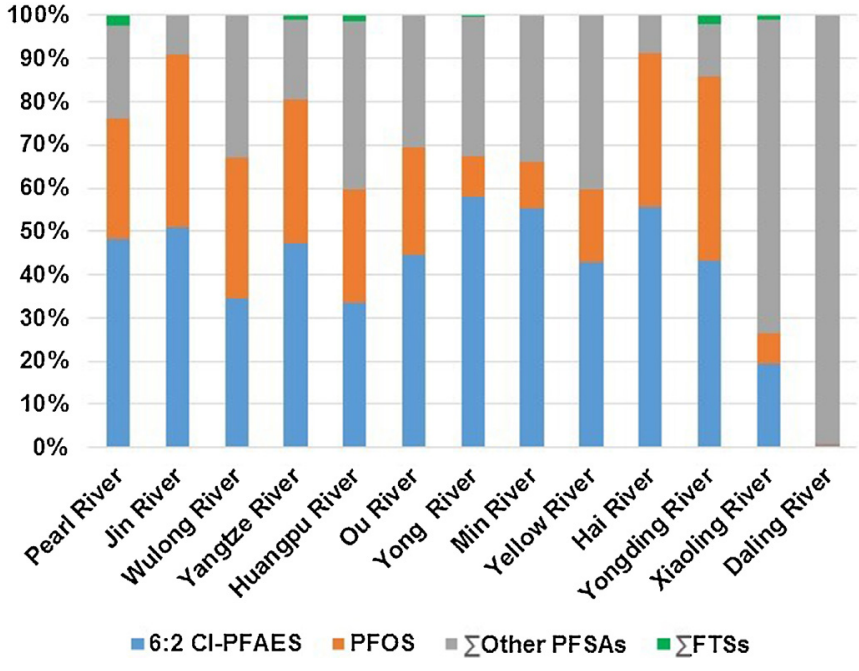

Fig. 5. Composition profiles of the identified fluoroalkyl sulfonates in riverine water samples quantified by the Orbitrap Tribrid HRMS method.

concentration of $0.2 \mathrm{ng} / \mathrm{L}$. 6:2 FTS had a low concentration of $0.2-0.4 \mathrm{ng} / \mathrm{L}$ (Table S5). Fig. 5 shows the proportion of $\sum$ PFSAs, $\sum 6: 2 \mathrm{Cl}$-PFAES and $\sum$ FTSs detected in thirteen river samples. Particularly, PFOS and 6:2 Cl-PFAES residue levels consisted of average proportions of $24 \%$ and $41 \%$ in the river samples, separately, indicating that 6:2 Cl-PFAES was widely used in China as an important PFSAs alternative, while the usage of FTSs was limited (average proportion: $1 \%$ ).

PFBS, PFHxS, L-PFOS, 6:2 Cl-PFAES were detected in all samples by LC-MS/MS method at concentrations in the range of $0.5-424 \mathrm{ng} / \mathrm{L}, \quad 0.3-16.7 \mathrm{ng} / \mathrm{L}, 1.9-14.2 \mathrm{ng} / \mathrm{L}$ and $1.9-40.2 \mathrm{ng} / \mathrm{L}$ respectively. PFDS was also only found in Yellow river at a low concentration of $0.2 \mathrm{ng} / \mathrm{L}$. 6:2 FTS was also detected in several samples ranging $0.1-0.3 \mathrm{ng} / \mathrm{L}$, close to the MQL (Table S5). The quantitative results of LC-Orbitrap Tribrid HRMS showed a good correlation with LC-MS/MS $(R>0.999$, Fig. S6), indicating Orbitrap Tribrid HRMS could be used as a reliable quantitative strategy in trace analysis of the fluoroalkyl analogs.

Additionally, the SPE procedure was a general method for retreatment of most poly- and perfluoroalkyl substances in water samples. The retrospective characteristic of the full scan $\mathrm{MS}^{1} / \mathrm{dd}-$ $\mathrm{MS}^{2}$ data analysis was also used for suspect screening and identification of other emerging fluorinated compounds such as perfluoroalkyl carboxylates and novel Cl-PFAES analogs [40].

\section{Conclusions}

A new method based on SPE-LC-Orbitrap Tribrid HRMS was established for simultaneous qualitative and quantitative analysis of PFSAs, FTSs and the emerging 6:2 Cl-PFAES. Good results on selectivity, linearity, MDL, trueness, precision, recovery and matrix effect were obtained. Compared with the routine LC-MS/MS quantification strategy, the Orbitrap Tribrid HRMS method showed comparable linear dynamic range, sensitivity and better selectivity. Moreover, the high mass resolution characteristic of Orbitrap Tribrid HRMS also provided specific advantages on accurate determination of PFSA analogs due to consistent instrument response of linear and branched PFOS isomers as well as on elimination of plausible environmental interferents such as TDCA. Further application of the Orbitrap quantitative method are warranted to in-depth evaluation on the reliability of the method for various kinds of environment matrices.

\section{Acknowledgments}

This work was jointly supported by the National Basic Research Program of China (2015CB453102) and National Natural Science Foundation (21361140359, 21207140).

\section{Appendix A. Supplementary data}

Supplementary data associated with this article can be found, in the online version, at http://dx.doi.org/10.1016/j.chroma.2016.01. 039.

\section{References}

[1] E. Kissa, Fluorinated surfactants and repellents, CRC Press, 2001.

[2] R.C. Buck, J. Franklin, U. Berger, J.M. Conder, I.T. Cousins, P. de Voogt, A.A Jensen, K. Kannan, S.A. Mabury, S.P.J. van Leeuwen, Perfluoroalkyl and polyfluoroalkyl substances in the environment: terminology, classification, and origins, Integr. Environ. Assess. Manag. 7 (2011) 513-541.

[3] S.H. Fang, X.W. Chen, S.Y. Zhao, Y.F. Zhang, W.W. Jiang, L.P. Yang, L.Y. Zhu, Trophic magnification and isomer fractionation of perfluoroalkyl substances in the food web of Taihu Lake, China, Environ. Sci. Technol. 48 (2014) 2173-2182.

[4] Z. Lu, L. Song, Z. Zhao, Y. Ma, J. Wang, H. Yang, H. Ma, M. Cai, G. Codling, R. Ebinghaus, Z. Xie, J.P. Giesy, Occurrence and trends in concentrations of perfluoroalkyl substances (PFASs) in surface waters of eastern China, Chemosphere 119 (2015) 820-827.

[5] B. Gonzalez-Gaya, J. Dachs, J.L. Roscales, G. Caballero, B. Jimenez, Perfluoroalkylated substances in the global tropical and subtropical surface oceans, Environ. Sci. Technol. 48 (2014) 13076-13084

[6] X. Wang, C. Halsall, G. Codling, Z. Xie, B. Xu, Z. Zhao, Y. Xue, R. Ebinghaus, K.C. Jones, Accumulation of perfluoroalkyl compounds in Tibetan mountain snow: temporal patterns from 1980 to 2010, Environ. Sci. Technol. 48 (2014) $173-181$.

[7] J. Campo, F. Perez, A. Masia, Y. Pico, M. Farre, D. Barcelo, Perfluoroalkyl substance contamination of the Llobregat River ecosystem (Mediterranean area, NE Spain), Sci. Total Environ. 503-504 (2015) 48-57.

[8] The 9 new POPs: An introduction to the nine chemicals added to the Stockholm Convention by the Conference of the Parties at its fourth meeting, Stockholm Convention on Persistent Organic Pollutants (POPs) (2010) 14

[9] S.W. Wang, J. Huang, Y. Yang, Y.M. Hui, Y.X. Ge, T. Larssen, G. Yu, S.B. Deng, B. Wang, C. Harman, First report of a Chinese PFOS alternative overlooked for 30 years: its toxicity persistence, and presence in the environment, Environ. Sci. Technol. 47 (2013) 10163-10170.

[10] E.F. Houtz, C.P. Higgins, J.A. Field, D.L. Sedlak, Persistence of perfluoroalkyl acid precursors in AFFF-impacted groundwater and soil, Environ. Sci. Technol. 47 (2013) 8187-8195.

[11] A. Jahnke, U. Berger, Trace analysis of per- and polyfluorinated alkyl substances in various matrices-how do current methods perform? J Chromatogr. A 1216 (2009) 410-421.

[12] E.I.H. Loi, L.W.Y. Yeung, S.A. Mabury, P.K.S. Lam, Detection of commercial fluorosurfactants in Hong Kong marine environment and human blood: a pilot study, Environ. Sci. Technol. 47 (2013) 4677-4685.

[13] R. Vestergren, S. Ullah, I.T. Cousins, U. Berger, A matrix effect-free method for reliable quantification of perfluoroalkyl carboxulic acids and perfluoroalkane sulfonic acids at low parts per trillion levels in dietary samples, J. Chromatogr. A 1237 (2012) 64-71.

[14] M. Takino, S. Daishima, T. Nakahara, Determination of perfluorooctane sulfonate in river water by liquid chromatography/atmospheric pressure photoionization mass spectrometry by automated on-line extraction using turbulent flow chromatography, Rapid Commun. Mass Spectrom. 17 (2003) 383-390.

[15] M. Villagrasa, M. Lopez de Alda, D. Barcelo, Environmental analysis of fluorinated alkyl substances by liquid chromatography-(tandem) mass spectrometry: a review, Anal. Bioanal. Chem. 386 (2006) 953-972.

[16] J.W. Martin, K. Kannan, U. Berger, P.D. Voogt, J. Field, J. Franklin, J.P. Giesy, T. Harner, D.C.G. Muir, B. Scott, M. Kaiser, U. Järnberg, K.C. Jones, S.A. Mabury, H. Schroeder, M. Simcik, C. Sottani, B.V. Bavel, A. Kärrman, G. Lindström, S.V. Leeuwen, Peer reviewed: analytical challenges hamper perfluoroalkyl research, Environ. Sci. Technol. 38 (2004) 248A-255A.

[17] I. Langlois, U. Berger, Z. Zencak, M. Oehme, Mass spectral studies of perfluorooctane sulfonate derivatives separated by high-resolution gas chromatography, Rapid Commun. Mass Spectrom. 21 (2007) 3547-3553.

[18] T. Ruan, Y. Lin, T. Wang, G. Jiang, N. Wang, Methodology for studying biotransformation of polyfluoroalkyl precursors in the environment TrAC-Trend Anal. Chem. 67 (2015) 167-178.

[19] H.B. Jin, Y.F. Zhang, L.Y. Zhu, J.W. Martin, Isomer profiles of perfluoroalkyl substances in water and soil surrounding a Chinese fluorochemical manufacturing park, Environ. Sci. Technol. 49 (2015) 4946-4954.

[20] A. Rotander, A. Karrman, L.M. Toms, M. Kay, J.F. Mueller, M.J. Gomez Ramos, Novel fluorinated surfactants tentatively identified in firefighters using liquid 
chromatography quadrupole time-of-flight tandem mass spectrometry and a case-control approach, Environ. Sci. Technol. 49 (2015) 2434-2442.

[21] Y. Picó, M. Farré, D. Barceló, Quantitative profiling of perfluoroalkyl substances by ultrahigh-performance liquid chromatography and hybrid quadrupole time-of-flight mass spectrometry, Anal. Bioanal. Chem. 407 (2015) 4247-4259.

[22] C. Coscolla, N. Leon, A. Pastor, V. Yusa, Combined target and post-run target strategy for a comprehensive analysis of pesticides in ambient air using liquid chromatography-Orbitrap high resolution mass spectrometry, J. Chromatogr. A 1368 (2014) 132-142.

[23] K. George, U. Vincent, C. von Holst, Analysis of antimicrobial agents in pig feed by liquid chromatography coupled to orbitrap mass spectrometry, J. Chromatogr. A 1293 (2013) 60-74.

[24] L. Bijlsma, E. Emke, F. Hernandez, P. de Voogt, Performance of the linear ion trap Orbitrap mass analyzer for qualitative and quantitative analysis of drugs of abuse and relevant metabolites in sewage water, Anal. Chim. Acta 768 (2013) 102-110.

[25] J.V. Bussche, A. Decloedt, L. Van Meulebroek, N. De Clercq, S. Lock, J. Stahl-Zeng, L. Vanhaecke, A novel approach to the quantitative detection of anabolic steroids in bovine muscle tissue by means of a hybrid quadrupole time-of-flight-mass spectrometry instrument, J. Chromatogr. A 1360 (2014) 229-239.

[26] M. Farré, Y. Picó, D. Barceló, Application of ultra-high pressure liquid chromatography linear ion-trap orbitrap to qualitative and quantitative assessment of pesticide residues, J. Chromatogr. A 1328 (2014) 66-79.

[27] M.W. Senko, P.M. Remes, J.D. Canterbury, R. Mathur, Q. Song, S.M. Eliuk, C. Mullen, L. Earley, M. Hardman, J.D. Blethrow, H. Bui, A. Specht, O. Lange, E. Denisov, A. Makarov, S. Horning, V. Zabrouskov, Novel parallelized quadrupole/linear ion trap/orbitrap tribrid mass spectrometer improving proteome coverage and peptide identification rates, Anal. Chem. 85 (2013) $11710-11714$.

[28] S. Taniyasu, K. Kannan, M.K. So, A. Gulkowska, E. Sinclair, T. Okazawa, N. Yamashita, Analysis of fluorotelomer alcohols fluorotelomer acids, and shortand long-chain perfluorinated acids in water and biota, J. Chromatogr. A 1093 (2005) 89-97.

[29] Z. Zhou, Y. Liang, Y. Shi, L. Xu, Y. Cai, Occurrence and transport of perfluoroalkyl acids (PFAAs), including short-chain PFAAs in Tangxun Lake, China, Environ. Sci. Technol. 47 (2013) 9249-9257.
[30] U.S. EPA., Compendium Method TO-1: Method for The Determination of Volatile Organic Compounds in Ambient Air Using Tenax ${ }^{\circledR}$ Adsorption and Gas Chromatography/Mass Spectrometry, U.S. EPA. (1984).

[31] T. Ruan, Y. Wang, Q. Zhang, L. Ding, P. Wang, G. Qu, C. Wang, T. Wang, G. Jiang, Trace determination of airborne polyfluorinated iodine alkanes using multisorbent thermal desorption/gas chromatography/high resolution mass spectrometry, J. Chromatogr. A 1217 (2010) 4439-4447.

[32] P.A. Lyon, K.B. Tomer, M.L. Gross, Fast atom bombardment and tandem mass spectrometry for characterizing fluoroalkanesulfonates, Anal. Chem. 57 (1985) 2984-2989.

[33] G. Arsenault, A. McAlees, R. McCrindle, N. Riddell, Analysis of perfluoroalkyl anion fragmentation pathways for perfluoroalkyl carboxylates and sulfonates during liquid chromatography/tandem mass spectrometry: evidence for fluorine migration prior to secondary and tertiary fragmentation, Rapid Commun. Mass Spectrum. 21 (2007) 3803-3814.

[34] S.P. van Leeuwen, J. de Boer, Extraction and clean-up strategies for the analysis of poly- and perfluoroalkyl substances in environmental and human matrices, J. Chromatogr. A 1153 (2007) 172-185.

[35] N. Yamashita, K. Kannan, S. Taniyasu, Y. Horii, T. Okazawa, G. Petrick, T. Gamo, Analysis of perfluorinated acids at parts-per-quadrillion levels in seawater using liquid chromatography-tandem mass spectrometry, Environ. Sci. Technol. 38 (2004) 5522-5528.

[36] E. Commission, Commission Decision of 12 August 2002 implementing Council Directive 96/23/EC concerning the performance of analytical method and the interpretation of results., Off. J. Eur. Commun. L221 (2002) 8-36.

[37] E. Commission Council directive 96/23/EC of 29 April 1996 on measures to monitor certain substances and residues thereof in live animals and animal products and repealing Directives 85/358/EEC and 86/469/EEC and Decisions 89/187/EEC and 91/664/EEC Off. J. Eur. Commun. L125 (1996) 10-32.

[38] A. Ballesteros-Gomez, S. Rubio, S. van Leeuwen, Tetrahydrofuran-water extraction, in-line clean-up and selective liquid chromatography/tandem mass spectrometry for the quantitation of perfluorinated compounds in food at the low picogram per gram level, J. Chromatogr. A 1217 (2010) 5913-5921.

[39] A.O. De Silva, C. Spencer, B.F. Scott, S. Backus, D.C. Muir, Detection of a cyclic perfluorinated acid perfluoroethylcyclohexane sulfonate, in the Great Lakes of North America, Environ. Sci. Technol. 45 (2011) 8060-8066.

[40] T. Ruan, Y. Lin, T. Wang, R. Liu, G. Jiang, Identification of novel polyfluorinated ether sulfonates as PFOS alternatives in municipal sewage sludge in China, Environ. Sci. Technol. 49 (2015) 6519-6527. 